\title{
Les eaux troubles, figures de noyées dans l'œuvre de Jean Genet
}

\section{Diane Henneton}

\section{(2) OpenEdition}

1 Journals

\section{Édition électronique}

URL : http://journals.openedition.org/studifrancesi/9768

DOI : 10.4000/studifrancesi.9768

ISSN : 2421-5856

Éditeur

Rosenberg \& Sellier

\section{Édition imprimée}

Date de publication : 1 août 2017

Pagination : 270-287

ISSN : 0039-2944

\section{Référence électronique}

Diane Henneton, «Les eaux troubles, figures de noyées dans l'œuvre de Jean Genet », Studi Francesi [En ligne], 182 (LXI | II) | 2017, mis en ligne le 01 août 2018, consulté le 06 janvier 2021. URL : http:// journals.openedition.org/studifrancesi/9768; DOI : https://doi.org/10.4000/studifrancesi.9768

\section{(c)}

Studi Francesi è distribuita con Licenza Creative Commons Attribuzione - Non commerciale - Non opere derivate 4.0 Internazionale. 


\title{
Les eaux troubles, figures de noyées dans l'œuvre de Jean Genet
}

\begin{abstract}
This article analyses the drowning scenes in Jean Genet's work, focusing more specifically on the film script Mademoiselle and the play Les Paravents (The Screens). It argues that Leila's mysterious disappearance in The Screens is indeed a drowning scene and shows that the MademoiselleBruno couple in the film script (with the autobiographical content explained at the beginning of the article) and the Leila-Said pairing in the play are in fact a self-portrait of Jean Genet and his two mothers: one his biological mother, the other, Eugénie Régnier, his foster mother. As drowned figures are always feminine in Genet's work, all elements related to them are examined, including skirt, water, and darkness. These scenes reveal the writer's sadistic impulse toward the maternal figure, whom he dismisses in his texts, and show that this rejection of the mother figure creates space for his fantasy about being brought into the world through self-generation.
\end{abstract}

Deux noyées à proprement parler apparaissent dans l'œuvre de Jean Genet. L'une est l'institutrice du scénario Mademoiselle $e^{1}$, l'autre est une religieuse dans Miracle de la rose. Contrairement donc à toute attente, ce n'est pas dans le roman des marins, Querelle de Brest, que la noyade a lieu, mais dans deux œuvres qui évoquent l'enfance et l'adolescence de l'auteur. Et ce sont des noyées au féminin car seules les femmes chez Genet sont vouées à la disparition dans la profondeur des eaux dormantes.

Mais n'y a-t-il vraiment que deux noyées dans toute l'œuvre de l'auteur de NotreDame-des-Fleurs?

Après une présentation de la nature autobiographique de Mademoiselle et de la dimension maternelle de son héroïne, cet article s'attachera à mettre en évidence l'existence d'une troisième figure de noyée dans la pièce Les Paravents avant d'analyser les éléments clés des scènes récurrentes de noyade: jupe, obscurité et eau. Même si la disparition de la nonne dans Miracle de la rose est évoquée dans cet article, c'est essentiellement dans le scénario et la pièce de théâtre que ce thème est étudié en lien avec les couples Mademoiselle-Bruno et Leïla-Saïd. En dernier lieu, une interprétation de la noyade liée à la véritable nature de ces figures féminines sera proposée.

\section{Le matériau autobiographique dans "Mademoiselle"}

Le scénario Mademoiselle n'ayant été publié qu'en italien² en voici le résumé: la vie d'un petit village situé en bordure de forêt est troublée par une série d'actes cri-

(1) Mademoiselle, tapuscrit, archives François Truffaut. Il existe, à notre connaissance, deux autres versions du script, mais qui ne varient de celle-ci que par quelques détails. L’une est conservée chez Gallimard, l'autre dans le fonds Jean Genet de l'Imec.

(2) S. Colomba, A. Dichy, L'Immoralità leggendaria. Il teatro di Jean Genet, Roma, Ubulibri, 1990. Précisons ici que le présent article s'attache uniquement au scénario et non au film de Tony Richardson qui a pris quelques libertés avec le script de Jean Genet. 
minels (inondation, incendie et empoisonnement de l'eau) qui coïncide avec l'arrivée de deux travailleurs polonais, Manon et Anton, dont la scierie se situe dans une clairière proche. Anton dort sur place tandis que Manon s'est installé dans le village avec son fils Bruno qui est scolarisé. Bruno nourrit un amour secret pour son institutrice, «Mademoiselle», et essaie désespérément de gagner son affection. Mais celle-ci est attirée par le père qui ne semble pas faire grand cas d'elle et se forge dans le village une réputation de coureur de jupons. À chaque fois qu'elle le voit en compagnie d'une femme du village, Mademoiselle s'en aigrit un peu plus et multiplie les vexations infligées à l'enfant dont elle stigmatise les origines. Comme les actes criminels se multiplient et que l'enquête menée par les gendarmes piétine, la tension monte et les paysans accusent sourdement Manon dont le succès auprès des femmes et l'héroïsme manifesté lors des différents sinistres irritent les hommes du village. Cependant, Mademoiselle que personne ne soupçonne, perpétue ses crimes: la nuit venue, alors que le village est endormi, elle s'habille et se maquille devant un miroir où elle semble moins se contempler que se perdre dans ses pensées, puis sort mettre ses plans à exécution. Après un nouveau forfait, elle se donne à Manon rencontré dans la forêt environnante. Pendant ce temps, l'exaspération est à son comble dans la commune et les villageois organisent une véritable chasse à l'homme. Pris au piège dans son propre jardin, Manon succombera sous les coups des paysans, laissant derrière lui un orphelin qu'Anton décide d'emmener dans ses pérégrinations. Mais avant son départ, Bruno veut faire un dernier tour dans la forêt où le hasard lui fait rencontrer l'institutrice dont il a percé à jour la culpabilité. Celle-ci effrayée par l'enfant qui marche sur elle, recule et tombe par accident dans le lac où elle se noie.

Le scénario qui a été réalisé en 1966 par Tony Richardson a pour cadre un petit village situé à proximité d'une forêt et dans lequel on peut reconnaître Alligny-enMorvan où l'écrivain vécut de 1911 à 1923 après avoir été abandonné par sa mère moins d'un an après sa naissance et confié par l'Assistance publique à une famille qui l'a élevé jusqu'à ses 13 ans, âge légal auquel l'administration plaçait ses pupilles en apprentissage. Les précisions topographiques sur Alligny données dans Jean Genet, matricule 192.102 recoupent les indications du scénario ${ }^{3}$. Le village où se situe l'action de Mademoiselle se trouve en bordure de forêt, dans une sorte de cuvette. Or dans leur enquête sur le village où l'écrivain a grandi, Albert Dichy et Pascal Fouché précisent que la forêt borde la commune d'Alligny située au creux de la vallée du Ternin. Dans son script encore, Genet fait évoluer ses personnages autour d'un lac qui se situe un peu à l'écart du village comme l'étang d'Alligny au bord duquel, selon l'un de ses camarades d'enfance, l'écrivain passait des heures à lire et à rêvasser. Ces eaux dormantes berçaient peut-être, tout en l'entretenant, sa mélancolie. Enfin, l'un des lieux privilégiés du scénario est l'école-mairie au premier étage de laquelle habite l'institutrice. Or, à Alligny, le même bâtiment abritait l'école et la mairie au premier étage duquel étaient logés Pierre et Joséphine Choppart, instituteurs. La maison des parents nourriciers de Genet jouxtait l'école et comprenait parmi ses dépendances un bûcher que l'on retrouve dans le script comme étant celui de l'institutrice. Dans le projet de film, Bruno y empile les bûches tandis que Culafroy dans une autre œuvre autobiographique de Genet, Notre-Dame-des-Fleurs, y faisait des pointes. Quant aux personnages du scénario, il semble qu'ils ont été inspirés, pour une bonne part, par les habitants du village où Genet a grandi. Il y a en particulier l'institutrice dont Bruno est secrètement amoureux comme Genet l'a peut-être été de Joséphine Chop-

(3) A. Dichy, P. Fouché, Jean Genet, matricule 192.102, Paris, Gallimard, 2010, pp. 37-41. 
part qui fut à en croire le narrateur de Notre-Dame-des-Fleurs, l'une des «fées» de son enfance. S'il était besoin de démontrer encore l'importance des réminiscences d'Alligny-en-Morvan dans Mademoiselle, le nom véritable (cité dans la version Gallimard du script) de Joseph Guilleminot, le «svelte boucher à la moustache aiguë» d'Alligny suffirait à lui seul à désigner le village de l'auteur comme matériau premier de cette construction.

De cette période de son enfance où il fut, de l'avis de ses anciens camarades, choyé par sa mère nourricière, Genet dresse un tableau sombre, dominé par le sentiment qu'il a d'être exclu, d'être un étranger au sein du village. Cette blessure ouverte dans son enfance le tourmenta toute son existence. Dans sa dernière œuvre, Un captif amoureux, il notait: «La France où, entre six et huit ans, je me sentis étranger [...]» ${ }^{4}$, réflexion qu'il avait déjà faite lors d'un entretien avec Hubert Fichte: «[...] j'ai su très jeune que je n'étais pas français, que je n'appartenais pas au village» ${ }^{5}$. Ce sentiment, Genet le transpose dans Mademoiselle en se représentant sous les traits de Bruno, un enfant Polonais dont la mère est morte et qui n'a que son père. Il est l'étranger que l'auteur se sentait être à son âge. Louis Cullaffroy, autre pupille de l'Assistance publique qui a vécu à Alligny dans les mêmes années que l'écrivain apporte ce témoignage reproduit dans Jean Genet, matricule 192.102: «Dès qu'il y avait quelque chose dans le pays, nous étions accusés tout de suite. S'il y avait un feu, par exemple - et c'était fréquent -, c'était tout de suite notre faute. On nous appelait, en patois du Morvan, les "metteux-de-feu" ${ }^{6}$. Dans le projet de film, alors que l'institutrice est en réalité la coupable, tout le village se ligue pour accuser les Polonais. Une véritable parenté existe donc entre Genet et son personnage, Bruno; entre son enfance à Alligny et celle de Bruno. Une précision d'ordre vestimentaire vient encore confirmer que l'auteur mettait bien de lui-même dans son personnage: ce sont les culottes courtes qui servent de prétexte à Mademoiselle pour l'humilier devant les autres enfants. Or, dans le signalement de Genet paru dans le journal Le Briard suite à sa fugue de 1924, il est précisé que l'adolescent, quoique «assez grand pour son âge» portait des culottes courtes ${ }^{7}$, un détail qui l'a certainement gêné et qu'il se remémore au moment de la rédaction de son script.

\section{"Mademoiselle", une figure de la mère}

Plusieurs questions sont soulevées par les choix de l'auteur dans Mademoiselle: pourquoi, alors qu'il y avait un couple d'instituteurs dans son village, seule l'institutrice accède à la représentation dans son script? Pourquoi l'auteur transforme-t-il celle qu'il désignait dans Notre-Dame-des-Fleurs comme une fée en une criminelle qui fait preuve quelquefois d'affection, mais plus souvent de cruauté, à l'égard de Bruno? Comment expliquer enfin que c'est elle, et non Eugénie Regnier, qui représente la figure maternelle dans Mademoiselle?

Dans Jean Genet, matricule 192.102, Albert Dichy et Pascal Fouché citent le témoignage de Louis Cullaffroy qui révèle que Pierre Choppart, l'instituteur principal d'Alligny et voisin de la famille Regnier, s'étant pris d'intérêt pour le petit Genet,

(4) Un captif amoureux, Paris, Gallimard, 1986, p. 454.

(5) Entretien avec Hubert Fichte, L’Ennemi déclaré (éd. A. Dichy), Paris, Gallimard, 1991, p. 149.

(6) A. Dichy, P. Fouché, Jean Genet, matricule 192.102 cit., p. 98.

(7) Ibid., p. 115, note en bas de page.

(8) J. Genet, Notre-Dame-des-Fleurs, dans Euvres complétés, t. 2, Paris, Gallimard, 1966, p. 75. 
lui aurait donné des leçons particulières ${ }^{9}$. Or, alors que l'institutrice accède au rôle principal de son projet de film, Pierre Choppart, lui, disparaît totalement. Qui plus est dans le script, c'est Mademoiselle, et non lui, qui aide l'enfant à faire ses devoirs. On pourrait taxer Jean Genet d'ingratitude, mais il semble plutôt que ce parti pris obéisse à une logique consciente ou inconsciente du récit selon laquelle la figure maternelle doit être seule, sans enfants, sans mari, sans famille, c'est-à-dire disponible pour Bruno, alias Genet. C'est pour cette raison même que l'institutrice n'est pas désignée par son nom (qui ne sera jamais révélé dans le scénario, pas plus que son prénom d'ailleurs) mais par son statut de femme célibataire (mademoiselle).

Pourtant, cette mère que l'enfant désire ardemment et que l'adulte recherchera, à sa manière, toute sa vie, est représentée dans le scénario par une figure maternelle négative. Outre les crimes dont Mademoiselle se rend coupable et qui causent la vie à un villageois ainsi que la mort d'un nombre important d'animaux, elle est celle qui humilie l'enfant devant la classe. Or, Genet a subi une vexation publique à l'école primaire d'Alligny en Morvan. C'est lui-même qui en fit le récit dans un entretien avec Hubert Fichte réalisé en 1975, plus de 50 ans après les faits:

[...] je vous rappelle que je n'ai ni père ni mère, que j'ai été élevé par l'Assistance publique, que j'ai su très jeune que je n'étais pas français, que je n'appartenais pas au village. J'ai été élevé dans le Massif central. Je l'ai su d'une façon bête, niaise, comme ça: le maittre d'école avait demandé d'écrire une petite rédaction, chaque élève devant décrire sa maison, j'ai fait la description de ma maison; il s'est trouvé que ma description était, selon le maittre d'école, la plus jolie. Il l'a lue à haute voix et tout le monde s'est moqué de moi en disant: "Mais, c'est pas sa maison, c'est un enfant trouvé", et alors il y a eu un tel vide, un tel abaissement. J'étais immédiatement tellement étranger $[\ldots]^{10}$.

Une première remarque s'impose: selon le souvenir de l'écrivain, c'est l'instituteur, Pierre Choppart donc, qui donna ce sujet de rédaction aux enfants et lut le devoir de Jean Genet à la classe. Même si le choix d'un tel sujet était maladroit dans un village où une partie des écoliers étaient des enfants de l'Assistance publique, il n'y avait certainement pas d'intention maligne de la part d'un enseignant qui donnait de son temps pour aider Jean Genet dans son apprentissage, comme Louis Cullaffroy en a témoigné. Or, dans Mademoiselle, non seulement c'est la figure féminine de l'institutrice qui inflige cette blessure à l'enfant, mais elle le fait délibérément. Le sujet sur lequel porte l'humiliation de Bruno diffère de l'expérience vécue par l'auteur: dans le scénario, il s'agit des culottes courtes portées par l'écolier alors qu'il est déjà grand et devrait avoir des pantalons longs ${ }^{11}$. Mais dans son enfance Genet a ressenti plus qu'une mortification, un vide extrême, selon son témoignage, quand ses camarades de classe se sont moqués de lui alors qu'il avait fait la description de la maison où il vivait, la considérant comme sienne. L'extrême violence de son émotion à s'entendre dire que le foyer où il avait grandi n'était pas le sien, à s'entendre désigner comme un «enfant trouvé» le conduisit immédiatement à se sentir étranger, c'est à dire aussi d'une certaine manière à s'éloigner de ceux qu'il aimait. Dans son script, si le pantalon de Bruno déclenche la remarque de l'institutrice, celle-ci en profite pour le stigmatiser dans ses origines à plusieurs reprises devant toute la classe: «Et vous venez à l'école, débraillé, vêtu comme un vagabond. Comme un gitan. La veste

(9) A. Dichy, P. FouchÉ, Jean Genet, matricule 192.102 cit., p. 53.

(10) L'Ennemi déclaré cit., p. 149.

(11) Mademoiselle cit., p. 9, 33 et 58 (et même une fois, il est puni sans raison comme s'il était devenu le souffre-douleur de la maîtresse). 
sur l'épaule, comme un Polonais!» ${ }^{12}$. Et plus loin, elle prédit: «Vous ne serez jamais qu'un bohémien $[\ldots] \gg^{13}$. Ici, la fiction rejoint la réalité puisque dans le deuxième cas, c'est l'origine de l'enfant qui est stigmatisée ${ }^{14}$. Pourtant à un autre endroit du scénario, Mademoiselle fait preuve d'affection envers Bruno qu'elle aide à faire ses devoirs et encourage. Elle revêt alors des qualités maternelles auxquelles l'enfant est très sensible ${ }^{15}$. Ainsi donc, cette figure est double, à la fois tendre et dure, capable d'encourager l'écolier dans son apprentissage de l'orthographe, donc de l'écriture, ce qui n'est évidemment pas anodin pour un écrivain, mais aussi porteuse d'instincts destructeurs pour le narcissisme de l'enfant. Il y a donc en elle de la bonne et de la mauvaise mère comme si Genet avait réuni dans cette figure ses deux mères: celle qui, par son abandon, est responsable de son état d'enfant de l'Assistance publique stigmatisé par les autres dans son identité et celle qui lui prodigue attention et encouragements $^{16}$. Mais il semble que «la bonne mère» est impuissante à prendre le dessus sur l'autre. Un détail attire l'attention dans la scène où la maîtresse de Bruno l'aide à faire ses devoirs: c'est le mot sur lequel il fait une faute et qu'elle corrige: mourir, qu'il orthographie avec deux «r».

MLLE (regardant, penchée sur la table) - Où en es-tu? Très bien... Non regarde: mourir... mou-rir... un seul r. Tu vois?

Bruno leva la tête, et d'en bas, regarda Mlle avec tristesse.

BRUNO - Oui, il n'en faut qu'un. (un temps). Je crois que je ne m'y ferai jamais ${ }^{17}$.

C'est le seul mot du devoir de l'enfant qui est énoncé, ce qui n'est évidemment pas anodin. Il constitue une annonce de la mort qui attend Mademoiselle à la fin du scénario et cette découverte, même si les deux personnages n'en ont pas conscience les lie irrémédiablement. Bruno assistera d'ailleurs à l'accident au cours duquel elle perdra la vie. Mais l'enfant bute justement sur ce mot comme s'il refusait le sort qu'il indique. Une autre remarque s'impose ici: la faute de Bruno porte sur le nombre de «r»: il en met deux alors qu'il n'en faut qu'un. Le mot «mourrir» ainsi écrit par l'enfant est, à une lettre près (et si proche de l'autre: $M$ au lieu de N) le verbe «nourrir», comme s'il opposait une résistance inconsciente aux peurs qui l'habitent et échangeait une pulsion de vie contre une pulsion de mort. Dans le scénario, la mère de Bruno, comme nous l'avons signalé plus haut, est morte; celle de Genet aussi et même s'il l'ignorait dans son enfance, elle était, de par son abandon alors qu'il n'avait pas même atteint l'âge du sevrage, morte pour lui au sens où l'entend le psychanalyste André Green dans le chapitre intitulé «La mère morte» ${ }^{18}$. L’un et l'autre ayant déjà fait

(12) Ibid., p. 33

(13) Ibid., p. 45.

(14) Une autre scène d'humiliation relatée dans Journal du voleur, celle du tube de vaseline découvert par un officier de police dans la poche de Genet est, comme l'a finement remarqué GisèLE CHILD-OLMSTED dans Transfiguration of the Mother in Genet's «Journal du voleur» and «Un Captif amoureux», étroitement associée à la mère dans la confession de l'écrivain (The French Review, vol. 71, n. 1, pp. 46-47).

(15) Mademoiselle cit., pp. 42-43.

(16) Dans le livre qu'elle a consacré à Jean Genet, CARoline Daviron met en avant le visage de la mauvaise mère dans le couple formé par Ernestine et son fils (Elles, les femmes dans l'œeuvre de Jean Genet, Paris, L'Harmattan, 2007, pp. 136-137). GisÈLE CHILD-OLMSTED dans Transfiguration of the Mother in Genet's «Journal du voleur» and «Un Captif amoureux» (cit., p. 44) écrit que dans son œuvre, Genet fait alterner les figures de bonne et de mauvaise mère, mais selon nous, la bonne mère n'apparaît véritablement que dans le dernier livre de l'écrivain tandis que les autres sont parfois des personnages entièrement négatifs, parfois à mi-chemin entre les deux. Il s'agit donc moins d'une alternance des figures maternelles positives et négatives que d'une fusion des deux, ce qui rend l'identification de celles-ci particulièrement complexe.

(17) Mademoiselle cit., p. 43.

(18) A. GreEn, Narcissisme de vie, narcissisme de mort, Paris, éd. de Minuit, 1983, notamment pp. 231-232. 
l'expérience de la perte de la mère, ils trouvent une figure de substitution: c'est l'institutrice pour le personnage du script, et pour l'enfant Genet Eugénie Regnier, sa mère nourricière. Mais tous deux sont tourmentés par la même crainte: celle de connaître une nouvelle perte, ce qui, le travail des biographes de l'écrivain l'a révélé, s'est en effet produit. En 1922, Eugénie Regnier mourut subitement ${ }^{19}$, laissant Genet orphelin de mère une seconde fois. Mademoiselle, qui est, avec Notre-Dame-des-Fleurs, le seul texte connu dans lequel Genet évoque sa petite enfance dans le Morvan, livre un témoignage précieux de ses préoccupations et sentiments d'alors notamment envers la figure maternelle qu'il ne peut évoquer que de biais, par le truchement d'un tiers personnage. Il semble en effet que Genet ait extrêmement souffert de la perte de cette seconde mère. Selon Lucie Wirtz, enfant abandonnée et qui a été, tout comme Genet, élevée par les Regnier, les deux pupilles de l'Assistance publique considéraient Eugénie comme leur mère et celle-ci les traitait comme ses propres enfants. Lucie Wirtz et l'une de ses camarades d'enfance se souvenaient que sa disparition fut un véritable traumatisme pour le futur écrivain ${ }^{20}$. Mais plus encore que ces témoignages, ces quelques lignes de Genet dans un texte resté jusqu'à aujourd'hui inédit dit ce qu'elle a représenté dans sa vie: «Ma naissance eut lieu le jour de la mort d'une femme autoritaire qui me servait de mère: j'avais dix ans. Soudain détaché d'un arbre nourri de rêve, je chus. J'appris la blessante section des choses, leur discontinuité» ${ }^{21}$. Cette évocation aux accents bibliques et qui fait de la vie de l'enfant Genet auprès d'Eugénie Regnier un séjour édénique donne la mesure de son attachement à celleci. Remarquons ici qu'il décrit paradoxalement le jour de la disparition de celle-ci comme celui de sa naissance à lui, nous reviendrons sur cette idée plus bas ${ }^{22}$. Ainsi Genet ne pouvait pas donner à sa mère nourricière les traits qu'il a prêtés à l'institutrice, il ne pouvait pas faire de celle qui lui a prodigué la tendresse maternelle dont il était assoiffé un personnage capable de persécuter un enfant. Il y avait là une barrière qu'il n'a pas franchie. Et si à un moment donné dans l'œuvre une fusion s'est opérée entre les deux mères, c'est autour du motif de la disparition, disparition que dans son inconscient peut-être Genet avait besoin de reproduire pour tenter d'en finir avec son désir de retrouver la figure maternelle.

\section{Leïla et sœur Zoé, les deux autres noyées de l'œuvre genétienne}

Au tableau XIV des Paravents, Leilla disparait ${ }^{23}$. Cependant, à la différence des autres personnages du drame, elle ne réapparaitra pas derrière un paravent blanc pour en crever la surface et pénétrer au royaume d'Hadès où les autres protagonistes du drame, morts également, l'attendent. Pourtant, dans cette scène énigmatique elle se voit «descendre dans la mort». Où est-elle donc passée? de quelle mort meurt-elle exactement et pourquoi Kadidja déclare-t-elle dans les derniers instants du drame qu'elle ne reviendra pas? La didascalie finale de ce tableau se présente ainsi: «Elle [Leïla] s'enfonce définitivement dans sa robe qui était faite de façon-sorte de crinoline-qu'elle puisse, vers la fin, s'y noyer. La lumière revient sur scène» ${ }^{24}$. Plusieurs

(19) La cause de son décès n'est pas donnée par les biographes de Genet.

(20) Voir le témoignage de Marie-Louise Robert dans Jean Genet matricule 192103 cit., p. 89.

(21) J. GENET, manuscrit sans titre, fonds Jean Genet, Harry Ransom Research Center, p. 1.

(22) Voir dans la dernière partie de cet article le commentaire sur Rembrandt.

(23) J. Genet, Les Paravents, Paris, Gallimard, 1985, coll. «Folio», p. 223.

(24) Ibid. 
éléments ici sont à considérer au regard de deux autres scènes: l'une se situe dans Miracle de la rose, l'autre dans Mademoiselle:

Elle [Mademoiselle] poussa un cri sourd en tombant à la renverse. Bruno s'arrêta net, dans une position presque instable, un pied à peine posé. Il attendit un moment, puis, à pas lents, glacial, il s'approcha: il vit Mademoiselle dans l'eau comme une méduse, se débattant. Il esquissa le mouvement de se jeter à l'eau, mais il se ressaisit: Mademoiselle venait de couler. Elle s'enfonçait et elle avait cessé de se débattre ${ }^{25}$.

C'est dans Miracle de la rose, publié en 1946, que l'on trouve la première scène de noyade, il s'agit de la chute dans un bassin de sœur Zoé, l'une des religieuses de Mettray:

Daniel avait repris son service de clairon. Un matin qu'il se trouvait à sa place dans le Carré désert, près du bassin, afin d'être toujours prêt pour n'importe quelle sonnerie que lui commandait Guépin, sœur Zoé, qui allait de l'infirmerie à la chapelle entendre la messe basse, passa près de lui. La rage dut glacer le cœur de l'enfant. Il songea sans doute à son môme qui s'était coupé le doigt, à cause de la sœur, pour rester avec lui. Il dit bonjour à la sœur, criant: «Bonjour, sœur Zoé». Sorties de leur fonction, les religieuses étaient volontiers aimables. Elle répondit donc bonjour. Le clairon s'approcha d'elle et, quand ils furent l'un vers l'autre, ils étaient aussi très près du bassin. Le gamin vigoureux donna un coup d'épaule à la vieille qui, le souffle coupé, bascula dans la flotte. Ses jupes la soutinrent un instant, faisant d'elle un nénuphar énorme et ridicule, mais très vite elles se chargèrent d'eau et tirèrent au fond la religieuse muette d'épouvante et de honte. Le contact de l'eau sur ses jambes, ses cuisses, son ventre, la nouveauté d'un élément dont elle n'avait plus l'habitude, paralysaient la vierge. Elle n'osa ni un mouvement ni un cri. Elle s'enfonça. Il y eut encore un léger remous à la surface, puis ce fut le calme si pur de tous les matins d'avril. Sous les fleurs de marronnier, la vierge mourut noyée. L'enfant, d'un coup d'épaule, rajusta la bretelle rouge et blanche de son clairon, remit ses mains dans ses poches et, tranquille, lent, s'éloigna du bassin. Ce n'est que le lendemain qu'on découvrit le cadavre dans l'eau. Evidemment, on conclut à un accident, à un faux $\mathrm{pas}^{26}$.

Le verbe «s'enfoncer» utilisé dans les trois textes (pourtant écrits à de longs intervalles de distance) pour décrire la disparition du personnage, associé à "couler" dans le script et aux mouvements de descente et de remontée de Leïla qui sont intimement liés dans la pièce à un vocabulaire qui évoque l'univers marin ( «je reflotte à la surf...», le «roulis», le «creux... d'une vague») $)^{27}$ font de cette disparition de l'héroïne des Paravents une noyade, même si aucune eau à proprement parler, n'est présente. Mais il y a aussi, et surtout, la comparaison à un nénuphar pour la religieuse de Miracle de la rose dont les jupes flottent un moment à la surface du bassin et à une méduse pour décrire la noyade de Mademoiselle. Quel autre élément pourrait inspirer à l'écrivain une telle image si ce n'est les jupes de l'institutrice déployées dans l'eau du lac? Or, dans Les Paravents, Genet décrit la disparition de Leilla noyée dans l'ampleur de sa crinoline. Dans son imaginaire, il s'agit donc bien d'une descente au fond des eaux et les amples jupes qui flottent à la surface dans les trois textes sont l'indice du fil secret qui relie ces scènes dans son œuvre.

(25) Mademoiselle cit., p. 78.

(26) J. Genet, Miracle de la rose, Paris, Gallimard, 1990, pp. 312-313.

(27) Les Paravents cit., p. 223. 


\section{L'obscurité, la jupe et l'eau}

\section{L'obscurité}

Deux éléments présents dans les scènes de noyade et qui ont partie liée avec la figure maternelle seront commentés ici.

Examinons tout d'abord la présence de la nuit dans ces textes. Dans les dernières pages du script juste avant la chute de Mademoiselle dans le lac, Genet précise: «Mademoiselle, seule dans son appartement, faisait ses malles. Elle allait s'en aller dans quelques jours. Elle ne sortait plus, sauf très enveloppée de gaze, à la nuit tombante $[\ldots] \gg^{28}$. D'ailleurs, à chaque fois que l'institutrice apparaît devant son miroir se préparant à sortir pour commettre ses crimes, c'est ou bien le soir, ou bien une fois l'obscurité faite dans sa chambre ${ }^{29}$. Ailleurs, dans Les Paravents, outre l'ombre qui envahit la scène au moment de la disparition de l'héroïne au tableau XIV, le prénom même de celle-ci, Leilla, signifie la nuit ou plus exactement le crépuscule, en arabe. Cette ombre qui enveloppe les deux femmes est chargée tout à la fois de valeurs intimes, secrètes et inquiétantes.

Il ne suffit pas que le mot «nuit» soit un substantif féminin dans la langue française pour qu'il revête une charge puissamment maternelle, il faut donc en rechercher l'indice dans l'œuvre de l'écrivain et c'est la première version des Paravents qui le livre dans une étonnante comparaison: au tableau VII, en effet, la mère de Saïd déclare à son fils: «Je suis toute seule et la nuit est plate... (soudain solennelle) Mais non, la nuit s'est soulevée, elle s'est gonflée comme les mamelles d'une truie...» ${ }^{30}$. L'écrivain qui a grandi les treize premières années de sa vie à la campagne choisit cette image à dessein: une truie dont les mamelles se gonflent va mettre bas ou bien l'a déjà fait. La nuit dans son œuvre est donc bien maternelle. Une scène extraite d'Un captif amoureux le confirme. Il s'agit de celle au cours de laquelle Hamza étant parti au combat, Genet occupe sa chambre pendant la nuit. Allongé sur le lit, il écoute les bruits de la bataille qui se livre au dehors quand la mère de Hamza frappe à la porte et pénètre dans la pièce:

La lumière du ciel étoilé entra dans la chambre et derrière je distinguai une grande ombre. De façon à laisser croire que je dormais, je fermai les yeux à demi mais je voyais tout entre mes cils. Fut-elle dupe de ma ruse? La mère venait d'entrer. Venait-elle de la nuit, maintenant assourdissante, ou de cette nuit gelée que je porte avec moi en tous lieux?

\section{Et encore:}

Dès que nous fûmes sortis de Jordanie, l'image de Hamza avec sa mère ne quitta guère ma pensée. Cette image s'imposait d'une façon curieuse: je voyais Hamza seul, le fusil à la main, souriant et ébouriffé, tel qu'il m'apparut avec Khaled Abou Khaled, et sa silhouette ne se dessinait ni sur le ciel ni sur les façades des maisons, mais sur une grande ombre, une ombre que je peux dire épaisse, aussi étouffante qu'un nuage de suie dont les contours ou, comme disent les peintres, les valeurs, sculpteraient la forme lourde et immense de sa mère ${ }^{31}$.

La lourde silhouette de la mère apparaît donc comme «une grande ombre» qui trouve son origine dans la nuit, qu'elle soit extérieure à l'écrivain ou non. Une préci-

(28) Mademoiselle cit., p. 76. Souligné par nous. Une ligne plus bas, Genet précise encore: «C'était presque le soir».

(29) Ibid., p. 14, 21 et 58.

(30) Les Mères, tapuscrit, archives IMEC, p. 79. C'est nous qui soulignons.

(31) Un captif amoureux cit., p. 230 pour la première citation et p. 241 pour la seconde. 
sion dans le premier passage cité attire l'attention: cette nuit qui accompagne l'auteur en tous lieux est «gelée», tout comme l'eau dont la température saisit sœur Zoé se noyant dans Miracle de la rose, tout comme la mort. Dans le second passage, au lieu d'être rafraîchissante, cette ombre est étouffante comme un nuage de suie, une matière si proche des cendres qui évoquent aussi la crémation des morts.

\section{La jupe}

La jupe des trois noyées qui font l'objet de notre analyse possède une même caractéristique: elle est large tout comme celle d'Eugénie Régnier dont l'écrivain se remémore ailleurs les jupons blancs ornés de volants ${ }^{32}$. Il est à noter d'ailleurs que Genet n'évoque quasiment jamais sa mère naturelle de même que sa mère nourricière dans ses œuvres, ce qui rend toute mention de celles-ci d'autant plus significative. Dans Les Paravents, comme Leila le dit, c'est à sa jupe qu'il faudrait s'accrocher pour l'empêcher de sombrer tout à fait dans l'aventure où Saïd l'a entraînée $e^{33}$ et le vêtement qui flotte un moment à la surface est la dernière trace visible de celle-ci comme des deux autres noyées. Notons d'ailleurs que le souvenir des jupons de sa mère revient à Genet alors qu'il regarde le couple formé par une mère et son garçonnet probablement accroché aux jambes de celle-ci comme le font tous les enfants en bas âge dont les bras saisissent les pans de la jupe maternelle pour s'en envelopper, la tête seulement restant visible. Juste après la mention des jupons, l'auteur remarque: «Dans chaque enfant que je vois - mais j'en vois si peu - je cherche à retrouver celui que j'étais, à l'aimer pour ce que j'étais» ${ }^{34}$. Peut-être cette réflexion s'applique-t-elle avec la même justesse à la mère de l'enfant. Or, si la mention de sa mère par l'auteur est extrêmement rare, il faut remarquer encore que deux traits seulement de celle-ci sont évoqués dans l'ensemble de son œuvre: ses jupes et sa poitrine, deux éléments par lesquels elle apparaît non seulement femme mais puissamment maternelle, c'est-àdire comme un abri au sein duquel l'enfant peut trouver refuge (les jupes) et comme mère nourricière (le sein). L'un comme l'autre occupe dans son œuvre une fonction métonymique.

Mais cette mère rarement évoquée n'est pas Camille Gabrielle Genet qui a transmis son nom à l'écrivain et dont il ne pouvait se souvenir ayant été abandonné par cette dernière à l'âge de 7 mois, il s'agit en fait d'Eugénie Régnier, sa mère nourricière ${ }^{35}$. Parmi les deux photos de celle-ci publiées par Albert Dichy et Pascal Fouché dans Jean Genet, matricule 192.102, celle qui a été prise vers 1912 devant la menuiserie de Charles Regnier à Alligny-en-Morvan montre une femme ronde et vêtue d'amples jupes qui tombent, sous son tablier, jusqu'au sol; dans ses bras, le petit Jean Genet.

Mais la jupe, si elle est l'indice de cette mère qui l'a porté dans ses bras et nourri, n'a-t-elle pas aussi une face inquiétante?

À ce sujet deux précisions dans Les Paravents ont retenu notre attention: l'une concerne Leïla, l'autre Warda, la patronne de la maison de passe. À un degré certes moindre que Leilla, son visage aussi est dissimulé. Bien que la seconde soit une prostituée tandis que la première est la femme de Saïd, leur condition de paria les rapproche

(32) Notre-Dame-des-Fleurs cit., p. 195.

(33) Voir Les Paravents cit., p. 129.

(34) Notre-Dame-des-Fleurs cit., p. 195.

(35) Genet l'a presque effacée dans ses évocations de son enfance, mais elle apparaît à quelques occasions. Voir à ce sujet la notice de D. Henneton dans Dictionnaire Jean Genet, Paris, Honoré Champion, 2014 , pp. 578-580. Sartre et à sa suite, nombre de commentateurs de l'œuvre de Genet sont en quelque sorte tombés dans le piège tendu par l'écrivain en focalisant uniquement sur sa mère naturelle. 
car Leilla est aussi une voleuse qui se fait prendre et emprisonner au cours de la pièce ${ }^{36}$. Ce lien qui unit les deux femmes, même si elles ne se fréquentent pas, est apparent dans leur tenue vestimentaire qui, tout en étant différente, présente des caractéristiques identiques: Leilla en effet est invisible sous sa longue robe et sa cagoule qui ne laisse voir que sa bouche et ses yeux, Warda quant à elle, est couverte jusqu'aux pieds de vêtements lourds et dorés, à la manière des vierges espagnoles enveloppées d'un long manteau brodé d'or. De plus, elle porte un faux nez et un maquillage si épais qu'on la dirait «plâtrée». Bien que sa tête ne soit pas recouverte d'une cagoule mais seulement d'un voile, la silhouette de sœur Zoé, dans son habit de nonne présente aussi des affinités avec celles de Leilla et de Warda. Les trois femmes sont donc, à des degrés différents certes, des non visibles. Les indications concernant leurs jupes en particulier attirent l'attention: l'une est la didascalie citée au début du présent article et qui décrit la robe de Leïla au moment où elle disparaît, la deuxième est le vêtement de la religieuse déployé à la surface du bassin, la dernière est la mention d'un jupon rose de Warda «vaste comme une crinoline». Outre le fait qu'elle est la patronne du bordel, Warda qui n'est ni jeune ni provocante n'en est pas moins l'aimant, le pilier central autour duquel gravitent les hommes: prostituée, elle est l'objet de leur fascination, mais pas en tant que corps désirable qu'elle recouvre d'ailleurs d'un nombre

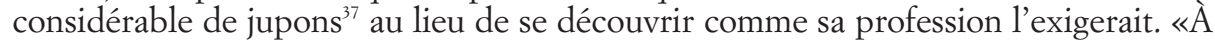
mesure que tu te nippes, à mesure que tu te plâtres, c'est toi qui recules et qui nous aimantes», déclare Mustapha, l'un des clients du bordel ${ }^{38}$. Cette attraction qu'elle exerce sur les hommes vient de ce qu'elle abrite la mort, justement sous ses jupes; elle l'avoue d'ailleurs sans détours à Mustapha qui en avait eu l'intuition:

WARDA: Mes toilettes! Dessous, il n'y a plus grand-chose...

MustaPHA, s'approchant d'un pas: S'il y avait la mort...

WARDA, l'arrêtant d'un geste: Elle y est. Tranquillement au travail ${ }^{39}$.

Une réflexion qu'elle se fait à elle-même un peu plus tôt dans la même scène confirme la nature morbide de son pouvoir tout en liant les vêtements avec l'ombre:

Warda, comptant ses bracelets: Il en manque un, tu l'apporteras. Je dois être lourde. (Un silence, et, comme pour elle-même) Manque un bracelet! Comme si j'étais un cercueil et qu'il manque un coup de marteau. (A Mustapha:) La nuit commence par l'babillage, la peinture. Quand le soleil est tombé, je ne pourrais rien faire sans mes parures... même écarter les jambes pour pisser je ne pourrais, mais juponnée d'or, je suis la Reine des Averses ${ }^{40}$.

Warda revêtue de ses habits amples et pesants se compare à un cercueil, c'est-àdire à un contenant qui abrite ou qui est destiné à accueillir un cadavre. Elle insiste par ailleurs sur la nécessité de ses parures une fois la nuit venue: «La nuit commence par l'habillage, la peinture $>^{41}$ dit-elle, des paroles qui font écho aux scènes de l'institutrice du scénario s'apprêtant avec soin devant son miroir avant de sortir la nuit pour commettre ses crimes. D'ailleurs, Mademoiselle, bien que son corps ne soit pas caché

(36) Elles sont peut-être encore liées dans la pensée de l'écrivain par le fait que les enfants de l'Assistance publique à Alligny étaient aussi appelés par les paysans «enfants de putain». Voir témoignage de Marc Kouscher in Jean Genet, matricule 192.102 cit., p. 101.

(37) Les Paravents cit., p. 30, elle déclare porter 3 jupons.

(38) Ibid., p. 33.

(39) Ibid.

(40) Ibid., p. 28. C'est nous qui soulignons.

(41) Ibid., p. 28. 
comme celui de Leilla ou de Warda, a aussi une forme d'opacité puisqu'elle n'a ni nom ni prénom dans le scénario et qu'on ne sait rien de son passé.

La jupe et le sein sont dotés d'une double polarité, positive et négative: quand l'écrivain évoque directement Eugénie Regnier, ils ont une polarité positive, rassurante, mais quand ces attributs sont donnés à d'autres figures maternelles, ils deviennent aisément menaçants. C'est le cas encore de la poitrine de l'institutrice dans Mademoiselle qui est un objet de désir pour Manon mais aussi le lieu où l'institutrice cache l'arsenic qu'elle va déverser dans l'abreuvoir ${ }^{42}$. Le sein nourricier devient alors un sein assassin qui n'est pas sans évoquer l'image de la petite bonne de Pompes funèbres au sein duquel le nourrisson décédé pend comme s’il avait reçu la mort même du lait maternel ${ }^{43}$.

\section{L'eau}

Un troisième élément est à considérer dans ces scènes: c'est l'eau. Ophélie, la mère de toutes les noyées en quelque sorte est convoquée par ces représentations, d'autant que Shakespeare précise que ses vêtements s'ouvrent lors de l'accident qui entraîne sa noyade et la portent un moment à la surface avant qu'elle ne s'enfonce dans les profondeurs d'une rivière boueuse. Une première remarque s'impose: l'héroïne malheureuse d'Hamlet disparaît dans une rivière, c'est-à-dire dans une eau courante tandis que chez Genet il s'agit d'une eau stagnante, fermée: un bassin pour sœur Zoé, un lac pour Mademoiselle. Quand à Leilla, elle s'enfonce dans sa robe comme dans un puits sombre. Nombreux sont les peintres, notamment au $\mathrm{XIX}^{\mathrm{e}}$ siècle, qui ont représenté Ophélie dans la position horizontale des corps flottants, comme endormie, bercée par les eaux. Chez Genet au contraire, les corps descendent à la verticale et les jupes sont tout ce qui persiste un instant des disparues, absorbées par l'élément liquide. Son eau, est intimement liée à l'ombre: c'est pendant la nuit que Mademoiselle disparaît dans le lac et le noir se fait sur scène lorsque Leïla s'enfonce, noyée dans ses jupes. Cette eau appartient à la catégorie qui boit au lieu d'être bue que Gaston Bachelard a identifiée dans L'Eau et les rêveries $d u \operatorname{repos}^{44}$. La nature mortifère de celle-ci apparaît nettement dans le scénario où elle tue à trois reprises: ce sont tout d'abord les eaux furieuses libérées quand l'institutrice ouvre les vannes et qui noient le bétail du village, puis l'eau empoisonnée par Mademoiselle qui avait caché un sachet d'arsenic entre ses seins, précise Genet, avant d'en vider le contenu dans l'abreuvoir, et enfin l'eau du lac où elle se noie à la fin du script. C'est une eau parfois coléreuse, parfois calme et qui loin de nourrir empoisonne et avale.

Mais revenons aux circonstances de ces noyades: dans l'œuvre de Shakespeare, Ophélie se noie par accident même si elle ne se débat pas, ce qui a fait dire à certains commentateurs qu'il s'agit d'un suicide. Il faudrait plutôt parler d'une mort consentie. Par ailleurs, elle est seule au moment où le drame se produit. Il n'y a donc personne susceptible de la secourir. Chez Genet, les trois noyades sont d'autant plus intéressantes qu'elles explorent trois mises en scène possibles de celle-ci. La première, qui

(42) Mademoiselle cit., p. 37.

(43) J. Genet, Pompes funèbres, Paris, Gallimard, 1989, pp. 129-130: «Sa douleur était plutôt physique, causée par cette amputation écœurante: la mort qui faisait tomber de votre sein le fardeau de chair qui s'y attachait par la bouche. Sa pensée écartait d'elle le souvenir de son enfant qui lui apparaissait comme un petit cadavre recroquevillé, accroché monstrueusement à l'un de ses nichons par les ongles et cette bouche morte».

(44) G. Bachelard, L'Eau et les rêves, Paris, Librairie José Corti, 1987, p. 77. 
date de 1943, si l'on considère le moment de la rédaction de Miracle de la rose, est la plus violente puisqu'il ne s'agit nullement d'un accident, mais d'un meurtre: celui de sœur Zoé par Daniel, un colon de Mettray qui la pousse dans le bassin par vengeance et assiste, impassible, à sa descente au fond des eaux.

La seconde, qui date de 1951, met en scène la noyade sous la forme d'un accident mais auquel l'enfant prend indirectement part puisque c'est sa marche menaçante en direction de l'institutrice qui est la cause de la chute de celle-ci dans le lac. Qui plus est, il hésite tant et si bien à la secourir qu'elle sombre avant qu'il n'ait pris une résolution.

Dans la troisième datée de 1958, la noyade est radicalement différente puisque Leilla, l'héroïne des Paravents est seule. La noyade n'a pas du tout les allures d'un accident, mais plutôt d'une mort pleinement acceptée. Au fil du temps donc, les scènes évoluent du meurtre à l'accident pour arriver à la mort consentie: Leilla se laisse volontiers guider dans sa descente vers l'au-delà.

Cette mort, qui chez Genet, est consubstantielle à l'eau est féminine. L'écrivain, nous l'avons souligné plus haut, a perdu deux mères: Camille Gabrielle Genet qui l'a abandonné et Eugénie Regnier qui l'a élevé jusqu'à l'âge de 12 ans, avant de mourir subitement, renouvelant en quelque sorte la malédiction du premier abandon. Ainsi l'enfance de Genet est doublement marquée par la mort de la mère. Dans Les Paravents d'ailleurs, l'hérö̈ne lors de sa descente dans la mort s'interroge: «[...] est-ce que la mort est une dame, une dame qui viendra me prendre[...]» $\gg^{45}$. Une remarque s'impose ici: la mort n'est pas imaginée conformément aux représentations traditionnelles sous forme de squelette, mais comme une «dame». Or, ce choix du mot «dame» plutôt que «femme» par exemple implique qu'il s'agit d'une femme d'âge mûr et inconnue, autrement dit une possible apparition de Camille Gabrielle Genet, cette «dame» dont l'écrivain n'a jamais su que le nom et dont il recherchera le visage jusque sur une inconnue dans la rue ${ }^{46}$. Certainement, enfant, il a espéré qu'elle viendrait le reprendre et l'arracher à son sort d'enfant abandonné. Cette figure maternelle tient aussi d'Eugénie Regnier dont les jupes restent gravées dans la mémoire de l'écrivain et flottent à la surface des eaux lourdes où s'enfoncent ses noyées comme un appel peut-être ou comme une nostalgie de l'abri maternel. De quelle mère s'agit-il en fin de compte? Cette «dame» n'est en réalité ni l'une ni l'autre mais la fusion des deux, unies dans ce qui leur est commun: la disparition, l'abandon de l'enfant Genet qui, en retour, les punit en quelque sorte en les livrant à l'eau. À cette impuissance qu'il a dû ressentir enfant, il oppose la toute-puissance de l'assassin qui ne subit pas le malheur, mais le provoque. Autrement dit, ce sont des pulsions sadiques qui s'expriment ici soit de manière très directe comme dans Miracle de la rose, soit sous le masque d'un accident ou encore dans la scène énigmatique des Paravents. On retrouve d'ailleurs ces pulsions dans les coups que Said voudrait infliger à Leilla ${ }^{47}$. L'aveu de ce désir de blesser la mère qui s'exprime à travers l'intention d'infliger des coups à Leïla est justement dévoilé dans un passage où il est immédiatement relayé par la confession du condamné à mort qui prend en charge la même pulsion, mais l'exprime dans sa forme la plus extrême, mêlant meurtre et amour envers la figure maternelle. On remarquera d'ailleurs que c'est par une blessure au ventre que le personnage aurait voulu commettre son crime:

(45) Les Paravents cit., p. 223.

(46) Voir plus bas la citation du Journal du voleur.

(47) Les Paravents cit., p. 222: «Saïd, mon bon Saïd, tu m’as éborgnée et tu as bien fait». 
LEÏLA: [...] Tu ne m'as jamais battue, Saïd?

SAÏD: Toutes les nuits je ne fais que m'entraîner. Dès ma sortie tu prends sur la gueule.

Un silence. On entend une voix qui parle.

La voIx du condamné à mort, très virile et décidée: Non. Si c'était à refaire: je m'approcherais de face, en souriant, et je lui offrirais une fleur artificielle, comme elle les aimait. Un iris en satin violet. Elle me remercierait. Aucune poupée blonde comme celles des films n'aurait écouté de pareilles foutaises que les miennes, et dites avec un si câlin sourire. C'est seulement...

LEÏLA, admirative: Qui c'est?

Le GARDIEN, grognon: Le condamné à mort. Il a tué sa mère.

La voIx du condamné à mort: ...quand mon discours aurait été fini, qu'elle aurait respiré la rose et qu'elle l'aurait piquée dans ses cheveux gris, que je lui aurais... ( $\grave{A}$ mesure la Voix s'exalte, et vers la fin elle psalmodiera et chantera.) délicatement ouvert le bide. Délicatement, j'aurais soulevé les rideaux du jupon pour regarder couler les boyaux, et j'aurais joué avec comme les doigts jouent avec les joyaux. Et la joie mon œil l'aurait redite à l'œil égaré de ma mère! $!^{48}$

L'eau assimilatrice dans laquelle Genet précipite ses noyées est une tentation pour l'enfant dans Mademoiselle où Bruno hésite un instant à plonger au secours de l'institutrice. Cette tentation est d'ailleurs clairement exprimée dans Querelle de Brest à propos de Madame Lysiane, figure maternelle du roman: «Il [Robert] laissait la maternelle féminité de cette femme forte et tendre à la fois l'envahir. Il nageait dans cet élément où parfois il était tenté de s'oublier» ${ }^{49}$. Cette mère de substitution que le personnage s'est trouvée est une eau dans laquelle il est possible de nager, de se fondre.

Comme nous l'avons signalé en début d'article, à la différence des autres morts des Paravents qui traversent une forêt et se retrouvent dans un au-delà qui accède à la représentation, Leilla s'enfonce dans une eau sombre d'où elle ne réapparaîtra jamais, entraînant d'ailleurs Saïd dans son sillage puisque ni l'un ni l'autre ne se joindra aux autres morts et que tous deux déclarent aller au «pays du monstre» ou du «dragon» ${ }^{50}$. Que peut bien être cette énigme du monstre, si ce n'est le couple mère-fils comme celui formé par Hamza et sa mère dans Un captif amoureux $x^{51}$ ? «Chacun des éléments a sa propre dissolution, la terre a sa poussière, le feu a sa fumée. L'eau dissout plus complètement. Elle nous aide à mourir totalement ${ }^{52}$ remarque Bachelard dans son essai. Cette eau fermée qui digère son mort, se l'assimile en quelque sorte, est un liquide amniotique qui renvoie l'être à sa vie prénatale: «Un grand nombre de rêves, souvent remplis d'angoisse, tels que ceux où l'on passe par des couloirs étroits, où l'on séjourne dans l'eau, reposent sur des fantasmes concernant la vie intra-utérine, le séjour dans le corps de la mère et l'acte même de naître» ${ }^{53}$. Cette association de la noyade avec la naissance est présente à l'esprit de Genet lorsqu'il évoque son origine dans La Sentence où il se décrit: «[...] fort de n'avoir jamais traversé une femme sauf à la minute de [s]a naissance, encore aveugle, muet, et sans doute encore noyés ${ }^{54}$.

(48) Ibid., pp. 129-130.

(49) Querelle de Brest, Paris, Gallimard, 1992, p. 103. C'est nous qui soulignons.

(50) Les Paravents cit., p. 167 pour Saïd et p. 176 pour Leila.

(51) Genet écrit en effet: «Finalement, je n'imaginai jamais une figure seule: toujours un couple dont l'une était prise dans l'attitude quotidienne et avec ses mensurations réelles, l'autre géante, simplement présente, ayant la consistance et les proportions d'une figure mythologique. Afin de résumer peut-être ce qu'était cette apparition: un groupe, couple-monstre dont une figure serait humaine, l'autre fabuleuse» (Un captif amoureux cit., p. 241).

(52) G. BACHELARD, L'Eau et les rêves cit., p. 125.

(53) S. Freud, L'Interprétation des rêves, Paris, PUF, 1987, p. 343.

(54) J. Genet, La Sentence, Paris, Gallimard, 2010, p. 28. Souligné par nous. 
Mais loin d'exprimer le seul désir de retour à l'état fotal de l'auteur, ce que les trois noyades dans l'œuvre de Genet indiquent c'est le meurtre symbolique de la mère qui est ainsi congédiée en quelque sorte par son fils. La première ligne de Querelle de Brest, dont la figure maternelle, comme nous l'avons montré plus haut, est une eau dans laquelle le personnage aspire à se baigner, est: «L'idée de meurtre évoque souvent l'idée de mer, de marins». Bien qu'il n'ait pas développé la question de la figure maternelle dans son article, Patrice Bougon remarque justement qu'il y a ici un jeu de mots et que là où l'auteur écrit «mer», il faut comprendre «mère» ${ }^{55}$. Ainsi Genet évoquerait d'une manière indirecte une préoccupation secrète: l'idée de meurtre évoque l'idée de mère. Cette dissolution voulue de la figure maternelle qui trouve son expression dans ces scènes de noyade, c'est aussi le choix d'une mort sans trace qui entre en adéquation avec la pensée d'un auteur qui se dit «issu d'une absence» dans La Sentence ${ }^{56}$.

\section{Le couple Saïd-Leila}

Si, comme nous l'avons montré précédemment, les indices de la figure maternelle: jupes, ombre et noyade sont associés au personnage de Leilla, c'est parce qu'en dépit de sa jeunesse et de l'existence d'un personnage désigné comme la mère de Saïd dans la pièce, elle est aussi une figure maternelle. Son invisibilité en est encore un indice: cachée sous sa cagoule, elle n'a pas de visage, tout comme Camille Gabrielle Genet n'en a jamais eu pour son fils qui n'a connu d'elle que son nom. Elle est plus une présence aux côtés du héros qu'une identité, le prénom même (la nuit) que lui donne Genet, ne fait que souligner ce qu'elle représente en fin de compte pour le héros:

Leïla: Où nous allons, Saïd?

Saïd: Où je vais, moi et moi tout seul, puisque tu n'es rien que mon malheur. À moins qu'en parlant de moi et de mon malheur, je dise nous. Eh bien, je vais et ça doit être loin, au pays du monstre. Que ça se trouve sous nos pieds, juste en dessous, où il n'y aura jamais de soleil, puisque je te porte et je te traîne t'es mon ombre $e^{57}$.

L'ombre dont nous avons identifié la nature éminemment maternelle est étroitement associée au mystère (le pays du monstre) et à l'invisibilité du personnage. Une fois emprisonné, Saïd dans sa conversation avec Leila lui reproche de ne pas l'avoir conseillé afin de lui éviter l'arrestation dont il a fait l'objet:

LEÏLA, d'une voix très douce: ....en marchant vite, et les reins courbés, mais surtout la veste pas boutonnée, personne n'aurait remarqué la bosse que faisaient les boîtes de conserves sous ta chemise.

SAIID, même douceur: Si tu as raison, c'est encore pire, puisque tu me montres le moyen de m'échapper une fois que c'est rendu impossible. Il fallait me prévenir avant...

LEİLA: J'étais déjà bouclée. Dans le cachot enfermé. Je ne pouvais plus te conseiller.

SAÏD: Je ne veux pas de conseils, mais tu peux me guider puisque tu es invisible et lointaine, derrière des murs épais... Et blancs... Et lisses... Et inaccessibles... Lointaine, invisible et inaccessible tu pouvais me guider ${ }^{58}$.

(55) P. Bougon, La Métaphore de la mer dans «Querelle de Brest», dans Toutes les images du langage. Jean Genet, sous la direction de F. Ekotto, A. Renaud et A. Vannouvong, Fasano, Schena Editore et Paris Alain Baudry et Cie Editeur, 2008, p. 21.

(56) J. Genet, La Sentence cit., p. 26.

(57) Les Paravents cit., p. 167.

(58) Ibid., pp. 121-122. Souligné par nous. 
Par le vol, la mendicité et l'épreuve de l'emprisonnement, Leïla se rapproche encore de la mère naturelle que Genet s'est imaginée dans Journal du voleur (à défaut de l'avoir connue) et à qui il prête un certain nombre de ses propres traits, la créant ainsi à son image:

[...] sous un réverbère, dans une rue de la ville où j'écris, le visage blafard d'une petite vieille, un visage plat et rond comme la lune, très pâle, dont je ne saurais dire s'il était triste ou hypocrite. Elle m'aborda, me dit qu'elle était très pauvre et me demanda un peu d'argent. La douceur de ce visage de poisson-lune me renseigna tout de suite: la vieille sortait de prison.

- C'est une voleuse, me dis-je. En m'éloignant d'elle une sorte de rêverie aiguë, vivant à l'intérieur de moi et non au bord de mon esprit, m'entraîna à penser que c'était peut-être ma mère que je venais de rencontrer. Je ne sais rien d'elle qui m'abandonna au berceau, mais j'espérai que c'était cette vieille voleuse qui mendiait la nuit.

- Si c'était elle? me dis-je en m'éloignant de la vieille. Ah! Si c'était elle, j’irais la couvrir de fleurs, de glaïeuls et de roses, et de baisers! J'irais pleurer de tendresse sur les yeux de ce poisson-lune, sur cette face ronde et sotte! Et pourquoi, me disais-je encore, pourquoi y pleurer? Il fallut peu de temps à mon esprit pour qu'il remplaçât ces marques habituelles de la tendresse par n'importe quel geste et même par les plus décriés, par les plus vils, que je chargeais de signifier autant que les baisers, ou les larmes, ou les fleurs.

- Je me contenterais de baver sur elle, pensais-je, débordant d'amour. (Le mot glaïeul prononcé plus haut appela-t-il le mot glaviaux?) De baver sur ses cheveux ou de vomir dans ses mains. Mais je l'adorerais cette voleuse qui est ma mère ${ }^{59}$.

Or, dans Journal du voleur, cette femme qui pourrait être sa mère, qui possède un visage rond et plat comme lui, qui sort de prison, vit tantôt de mendicité tantôt de ses vols tout comme Genet ${ }^{60}$, apparaît justement durant la nuit. Leïla, quant à elle, vole tout au long du drame à droite et à gauche (et est emprisonnée comme l'écrivain). Cachant ses larcins sous ses vêtements, elle rentre chez elle avec un gros ventre puis extrait un à un les objets de dessous ses jupes en leur parlant avec affection comme si elle les mettait au monde: «Leilla, sortant une râpe à fromage: Salut!... Je ne suis pas rancunière. Tu m'as râpé la peau du ventre, et pourtant je te salue. Tu vois un jour nouveau. Ici, c'est la maison de la Mère» ${ }^{61}$. Leilla est comme enceinte du vol et peut-être même du voleur. La mère, c'est autant Leïla que la mère désignée comme telle de Saïd. D'ailleurs, ce dernier se fait cette réflexion: «Si tu [Leilla] te colles à moi comme un morpion à une couille, comme la forme ronde colle à l'orange $[\ldots] \gg^{62}$ dans cette image de l'orange, il faut voir l'emboîtement, l'enveloppe ou l'utérus qui contient le fœtus, elle symbolise le fantasme d'involution dans le corps maternel. Un petit détail, mais qui a son importance, vient encore confirmer la nature secrète de Leîla: au moment où elle réapparaît sur scène avant de disparaître totalement dans sa crinoline, c'est-à-dire au moment où elle s'abandonne, en se plongeant dans l'élément liquide, à sa véritable nature - qui est maternelle -, elle porte des talons hauts, tout

(59) Journal du voleur, Paris, Gallimard, 1991, p. 22. (Souligné par nous). Dans le roman, l'auteur imagine qu'il vomirait dans les mains de sa mère retrouvée et l'héroïne des Paravents, quant à elle, se livrant à la mendicité pour mieux satisfaire Saïd remarque «(une mendiante)... pue. C'est son truc. On lui donne un sou et on se sauve en dégueulant» (Les Paravents cit., p. 134, souligné par nous).

(60) Au onzième tableau Leila semble avoir atteint une sorte d'apothéose en se livrant à la mendicité, activité grâce à laquelle elle gagne enfin le respect de Saïd:

«Leila, à voix douce: Saïd... maintenant je mendie comme il faut.

Said, admiratif: La chine! Et tu ne me le dis que cette nuit?» (Les Paravents cit., p. 127 et p. 134, il renouvelle son exclamation).

(61) Les Paravents cit., p. 95.

(62) Ibid., p. 168. 
comme la mère au début de la pièce à qui Saïd avait ordonné de mettre ses souliers à talons. Une fois qu'elle les avait chaussés, la mère apparaissait ainsi magnifiée aux yeux de son fils.

Saïd est en somme un héros qui a deux mères, comme l'écrivain lui-même. D'ailleurs, la pièce avait pour titre originel «Les Mères». Leilla serait donc une autre figure de la mère et Saïd le représentant de l'auteur dans la pièce. Genet n'a-t-il pas écrit dans «Comment jouer Les Bonnes»: «[...] je vais au théâtre afin de me voir, sur la scène (restitué en un seul personnage ou à l'aide d'un personnage multiple et sous forme de conte) $\gg^{63}$. Ainsi il a prêté à Saïd plusieurs caractéristiques qu'il s'est reconnues à lui-même ${ }^{64}$ : lâche, menteur et traître. Et que le personnage chargé de le représenter soit arabe - peuple que l'écrivain a toujours aimé - n'affecte en rien l'interprétation puisque, comme nous l'avons souligné plus haut, Genet s'est senti, dès l'enfance, étranger dans son village ${ }^{65}$. Par ailleurs, Saïd est un voleur peu habile qui, tout comme Genet, va de condamnation en condamnation ${ }^{66}$ de prison en prison ${ }^{67}$ et qui n'hésite pas à déclarer: «Je te le dis, je suis en train de devenir quelqu'un» ${ }^{68}$. Pourtant dans cette pièce, Genet brouille les cartes en distribuant les rôles quand il fait de Leilla l'épouse de Saïd. Mais dans ce drame, il n'y a pas d'union charnelle entre mari et femme, juste une communion d'âme à partir du moment où Leilla, incarcérée tout comme lui, apparaît à ses yeux comme un possible double féminin de lui-même. Un double féminin qui n'est autre que cette mère jamais vue que l'écrivain créé à son image et dont il fait une épouse pour le personnage qui le représente sur scène. Quelle plus complète fusion imaginer entre une mère et son fils? ${ }^{69}$

\section{Fantasme d'auto-engendrement}

Cette mère tellement désirée, pourquoi l'auteur la précipite-t-il ainsi dans des eaux où elle s'abîme? C'est qu'en fin de compte, elle lui sert plus morte que vivante car, au moment où il rédige ces œuvres, il n'est plus un enfant mais un artiste qui bâtit sur sa blessure pour reprendre les termes d'Hélène Cixous qui écrit: «Lui Genet chaque année naît et renaît de la blessure retrouvée, relue, sondée jusqu'au Souvenir $\gg^{70}$. Étoile dans le firmament, la mère chez Genet ne guide qu'à la condition d'être loin. Une fois emprisonné, Saïd accuse Leïla de ne pas l'avoir conseillé comme elle aurait dû et achève sa réplique par ses mots: «Lointaine, invisible et inaccessible, tu pouvais me guider» ${ }^{71}$.

(63) J. GEnET, «Comment jouer Les Bonnes», Les Bonnes, Paris, Gallimard, 1990, p. 10. Freud a identifié cette tendance des écrivains de la modernité littéraire à cliver leur moi en mois partiels comme manière de gérer l'éclatement du moi qu'ils ressentent. Voir «L'écrivain et le fantasme» in Névrose, psychose et perversion, Paris, PUF, 1973, pp. 220-221.

(64) J. GENET, L'Ennemi déclaré cit., p. 11.

(65) Voir note n. 5 en début d'article.

(66) Ibid., p. 81: Le Cadi à Saïd: «Ce que tu gagnes à chaque nouvelle condamnation, je vois, vaguement, où tu vas, mais moi, chaque condamnation me conduit vers quoi?».

(67) Ibid., p. 97, Leilla déclare: «Alors, au gnouf. Avec Saïd. Puisque maintenant on doit aller de village en village, autant aller de prison en prison... Avec Saïd».

(68) Ibid., pp. 169-170. Rappelons que cette pièce a été écrite alors que Genet avait déjà accédé à la notoriété.

(69) Le lien entre Madame Lysiane, Robert et son frère dans Querelle de Brest met en scène, de manière moins déguisée, la même préoccupation.

(70) Entretien de la blessure, Paris, Editions Galilée, 2011, p. 24. Et plus loin (p. 55): «Il fait du Traumatisme sa gelée royale, son régal amer».

(71) Les Paravents cit., p. 122. 
Mais dans son effort pour s'arracher à une fixation maternelle morbide, Genet découvre ceci: l'absence, la perte, aussi douloureuse soit-elle, libère et permet à l'artiste d'accéder à un autre niveau de création, elle en est peut-être même la condition. C'est la réflexion qu'il se fait devant la peinture de Ronde de nuit de Rembrandt et qu'il faut appliquer à sa propre œuvre: «Dès la mort de Saskia - je me demande s'il ne l'a pas tuée, d'une manière ou d'une autre, s'il ne s'est pas réjoui de sa mort - enfin son œil et sa main sont libres ${ }^{72}$, une idée toute sartrienne que Genet conduit un peu plus loin que l'auteur de L'Être et le néant (dans ce roman, Sartre écrit à propos de la disparition de son père: «La mort de Jean-Baptiste fut la grande affaire de ma vie: elle rendit ma mère à ses chaînes et me donna la liberté» ${ }^{73}$ ) en exprimant très directement une pulsion de meurtre associée au manque originel. La manière dont il maltraite les personnages de mère dans son œuvre en est un signe: par exemple le quasi-viol, sur la tombe de son enfant, de Juliette, la petite bonne de Pompes funèbres ${ }^{74}$. Mais l'expression la plus extrême de ce désir est dans Les Paravents celle du condamné à mort s'adressant à sa mère qu'il a assassinée:

La voix du condamné à mort: Madame, c'est pour ma liberté. Madame, je n'aime que votre ventre où pendant neuf mois j'ai pris la forme rose que la rose de votre matrice a laissé tomber sur le carreau comme une quenelle sur une assiette. Aujourd'hui, je me libère pour de bon de votre ventre trop chaud! je le refroidis ${ }^{75}$.

Le condamné à mort, c'est encore une autre figure de l'auteur dans la pièce. Sa confession, habilement entrelacée au dialogue de Saïd et Leïla détenus dans la même prison que lui, avoue de manière très crue, une pulsion matricide qui ne s'exprime chez Saïd que de manière déguisée, d'où la figure opaque, masquée de Leilla grâce à laquelle elle peut prendre forme. Ainsi, dans la nuit de la prison, la voix du condamné à mort est en quelque sorte la voix inconsciente de Saïd.

Ce désir de tuer la mère dans et par l'œuvre débouche sur un fantasme d'auto-engendrement que l'écrivain a exprimé à plusieurs reprises dans ses textes, dans Journal du voleur notamment qui doit lui servir de Genèse comme il le déclare à la dernière page de son livre, mais encore et surtout sans aucun fard dans La Sentence:

Avant de naître, avant que d'être avec ce corps, j'étais hors du temps et de l'espace, et c'est peut-être moi, ce fut mon impatience qui forcèrent l'homme et la femme, qui forcèrent la fornication un certain jour, choisissant moi-même le spermatozoïde et l'ovule, avec eux la position - latitude et longitude - d'où mon corps partirait afin de s'expatrier de cette partie du monde où le regard incongru de dieu se fait toujours plus menaçant. Cette divagation me laisse capter l'idée que mes géniteurs avaient besoin que je sois afin d'être eux-mêmes un moyen pour qu'ils me mettent au monde ${ }^{76}$.

Comme ce texte l'exprime, Genet veut se tenir lieu d'origine. Face au sentiment d'impuissance dans lequel l'abandon (et l'on peut même dire le double abandon puisque sa mère nourricière, Eugénie Regnier, est morte alors qu'il n'était encore

(72) Ce qui est resté d'un Rembrandt déchiré en petits carrés..., Paris, Gallimard, 1989, p. 25.

(73) J.-P. Sartre, Les Mots, Paris, Gallimard, 1964, p. 11. Et plus bas, il écrit: «Eût-il vécu, mon père se fût couché sur moi de tout son long, et m'eût écrasé. Par chance, il est mort en bas âge [...]».

(74) Pompes funèbres cit., pp. 195-196. Genet conclut la scène par ces mots: «Qu'on l'encule sur la tombe de sa fille, je serai content».

(75) Les Paravents cit., p. 134. On notera la volonté de désacraliser la mise au monde de l'enfant par le caractère grotesque de la comparaison choisie.

(76) La Sentence cit., p. 28. 
qu'un enfant) l'a laissé, il procède au meurtre symbolique de la mère, mais celui-ci a lieu dans l'eau, comme s'il voulait se donner le pouvoir de l'appeler à renaitre, ce qu'il fait, à sa manière, par son œuvre. Dans Les Paravents qui met en scène la troisième version de cette noyade, l'enfant devenu le mari (un mari qui observe la plus grande chasteté dans ses relations avec sa femme) la suit, même si c'est avec un certain retard, dans sa disparition au lieu de rester au bord de l'eau comme dans les versions précédentes de noyade de la figure maternelle. Mais si elle doit renaître par le pouvoir créateur de l'écrivain, cette mère sera à l'image du fils: voleuse et mendiante comme Leila dans Les Paravents. Genet renverse ainsi l'ordre originel: il confisque à la mère son pouvoir de mettre au monde et se l'approprie, se forgeant ainsi, livre après livre, une stature de démiurge, totalement maitre de son destin.

Peu étudié parce qu'encore inédit, le scénario de Jean Genet, Mademoiselle, a souffert aussi du mauvais accueil réservé au film de Tony Richardson. Pourtant ce script possède un intérêt réel, tant du point de vue cinématographique que thématique. Mise en corrélation avec Les Paravents et Miracle de la rose, la mort de l'institutrice dans ce script permet de proposer une interprétation de l'énigmatique scène de la disparition de Leïla dans la pièce et d'éclairer le motif de la noyade chez Jean Genet. Celle-ci, comme le présent article le montre, ne concerne que les figures maternelles qui prennent dans son œuvre différents visages mais qui sont intimement liées entre elles par l'eau et l'obscurité, ainsi que par la jupe qui est l'indice du lien autobiographique de celles-ci avec l'écrivain. Ces noyées qui réapparaissent dans son œuvre révèlent l'ambiguïté profonde des sentiments de l'auteur vis à vis de ses deux mères disparues trop tôt et montrent comment Jean Genet devenu adulte s'est construit en tant qu'écrivain sur ce manque qui a nourri sa création artistique et tracé la voie par laquelle il a, comme Saïd dans Les Paravents, trouvé son chant. Est-ce par une prescience toute rimbaldienne que Genet, malade, se sachant arrivé au bout de sa vie et ayant à peine achevé les dernières corrections d'Un captif amoureux prend un bain dans sa chambre d'hôtel, lui qui dans sa vieillesse avait horreur de se laver, comme s'il consentait à cette réunion finale avec l'élément liquide? Et, ultime coup du sort: ce dernier bain lui apporte la mort au moment même où il sort de l'eau. 